\title{
Multi-Scale Smart Building Simulation Platform for Energy, Market and Automation Solutions
}

\author{
Jean-Nicolas Louis Jari Pulkkinen \\ Water, Energy and Environmental Engineering, University of Oulu, Finland, \\ jean-nicolas.louis@oulu.fi, jari.pulkkinen@oulu.fi
}

\begin{abstract}
The increasing presence of smartness in buildings to increase the energy efficiency of these buildings makes it difficult to properly anticipate the impact of these technologies. The development of digital tool for creating residential load profile are prolific but their use, especially applied to smart buildings being able to combine both power and thermal energy management, are unavailable. This research presents a tool developed to integrate power and heat demand using a flexible topology to define buildings energy needs across time resolution. It also integrates small-scale renewable energies such as wind and solar to increase the production of local electricity. The entire model can be parametrised and personalised through its user interface. The model demonstrated robust simulation load profiles generation throughout all time resolutions
\end{abstract}

Keywords: smart buildings, high resolution, heat and power, demand response, real-time pricing

\section{Smart buildings}

The use of smart buildings and its role in shaping the new energy efficiency plans from the EU Green deal is crucial (EU Commission, 2019). The smart building is becoming a new standard in both the standardisation body and in the regulation with the implementation of the Smart Readiness Indicator (SRI) in EU. This comes in combination with the smart grid standards that are becoming more and more implemented in real-life microgrids, or in the establishment of positive energy district such as those built in frame of the EU lighthouse project Making-City. International research activities has put a strong focus on building flexibility and their role in integrating more distributed variable renewable energies onto the distribution network. Such research are built through the International Energy Agency (IEA) and its series of annex targeted on Energy in Buildings and Communities program (IEA-EBC) in its Annex67 (Jensen et al., 2017) and its future Annex82 on Energy Flexible Buildings.

Multi-energy vector (Electricity, heat, hydrogen, etc..(Orecchini and Santiangeli, 2011)) applied to building simulation and optimisation have been deployed for at least 20 years with for instance the doctoral thesis of Stokes (2005) on producing fine-grained load model to support low voltage power network. These models pro- duce load profiles for network performance analysis but are usually not publicly available, nor the code that goes along with the models. In more recent years, tool for simulating multi-scale simulation with medium horizons were developed and integrated Stokes' work into an opensource Excel VBA tool (McKenna and Thomson, 2016). Little by little, models are becoming more robust and integrate occupancy from statistics (Wang et al., 2018) or from appliance usage probability (Yilmaz et al., 2017). There is however a need for a new tool that combines latest development on the field of flexible energy and that is open source and accessible by all.

The Smart Building Model (SBuM) (Louis et al. 2016) was originally created to measure the impact of demand response and occupant behaviour on the building's energy performances using different levels of control. Controls included basic information-based system to raise awareness to full control over the appliances installed in the building. It has further been developed to integrate the thermal performance of buildings and integrate electric heating technologies as a demand response tool for smart building (Pulkkinen and Louis, 2019).

This work aims at developing an open and user-friendly tool to setup multi-scale building energy models, integrating variable time dependent demand response programmes and providing detailed load profiles that are representative of input profiles. The model shall be adaptable for multi-regional study and work under various weather conditions. The model outputs should cover detailed energy analysis, monetary impacts of different demand response rate, and account for the environmental impact of the related energy consumption.

\section{Heat and power model}

The SBuM model is a Markov-chain model developed using modules that are plugged in into the main model. It is composed of multiple modules that integrates the following: a small-scale energy production, control options for appliances and heating systems, and a home appliance model. Other modules are being developed to cover the full range of demand response possibility for smart building such as the domestic hot water. Unlike most mathematical model presented in the literature, the SBuM model is available open source (https://github. com/jeanlouisnico/SBuM) and can be run through 
the MatLab ${ }^{\mathrm{TM}}$ simulation platform.

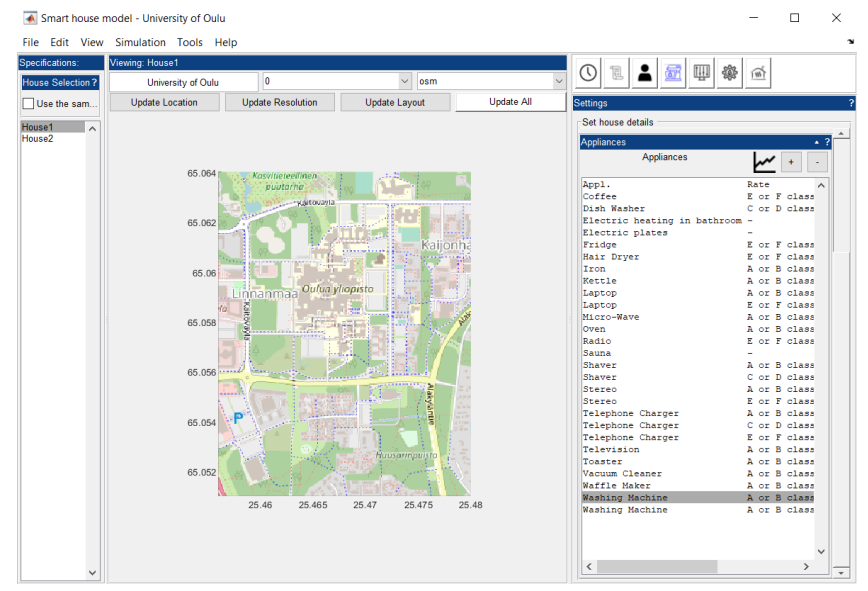

Figure 1. Smart Building Model (SBuM) interface seen from the MatLab ${ }^{\mathrm{TM}}$ simulation platform

Interface allows to have flexible and easy-to-use tuning of the building and all the variables considered in the SBuM tool are tunable. It allows as well to do reporting and retrieve all building characqteristics that are used as input variables to the model.

\subsection{Input data}

A set of input data are required to be able to run the model and use its possibilities. There are six (6) type of input data category needed to fill in and are visible on the righthand side of Figure 2: the weather files, the household description from appliances to users, the time resolution and horizon of the simulation, the optional power productions units, the type of electricity contracts used, and the building characteristics detailing its physical properties (see section 2.2.2).

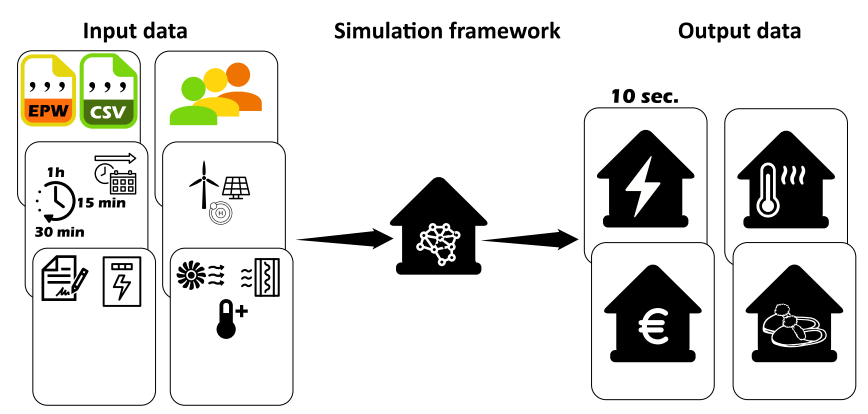

Figure 2. SBuM architecture from input data, modelling, and output variables.

\subsubsection{Climatic data}

The model is flexible on the type of weather format to be included and any EPW weather file can be given as an input to the model (Crawley et al., 2001). EPW files are available in open access ${ }^{1}$ and may be modified to represent

\footnotetext{
${ }^{1}$ http://climate.onebuilding.org
}

new weather for extreme conditions or represent future climatic conditions considering the different RCP scenarios and other General Circulation Model (GCM). Most of the data are gathered with an hourly time resolution, different weather data were linearly interpolated to match the simulation time-frame e.g. outdoor temperature, with some randomness in the interpolation e.g. solar radiation and wind speed.

\subsubsection{Households characteristics}

The SBuM model includes a set of variables that define the house to be modelled. All appliances that have a power signature may be included in the model and are characterised by their daily load profile probabilities, usage duration, weekly maximum usages, nominal power ratings, weekday/weekend distributions and control options for demand-side management implementation. The appliances characteristics and usage statistics are calibrated using the EuP reports, specifically adapted for Finland and the Finnish national statistics on energy use; they are however tunable to match any other performances when needed or reflect updated information compared to the previous EuP studies. For detailed information on households' specifics, refer to Louis et al. (2016). Similarly to Yilmaz et al. (2017), the occupancy distribution is deduced from appliances activities that are set in the beginning of the simulation. Their daily distributions are alterable to model any appliance distribution that the model needs to consider during the simulation.

\subsubsection{Small-scale production system}

Small-scale production units that can be utilised in the model include so far only solar photovoltaic panels, but experimental modules for wind turbines and fuel cells (Proton Exchange Membrane model) are also available. These models are somewhat simplified as multiple variables, such as the terrain and relief of the surroundings are not used in the model. The PV-model requires a set of 12 inputs characterising the photovoltaic modules. The methodology and the used equations are established from Luque and Hegedus (2010) and thus the made assumptions come from the book. The methodology consists calculating the voltage and current of one single cell of a solar module and then, depending on the solar irradiance, evaluating the power output of a series of cells. It is possible to outline real performance from technology available on the market.

\subsubsection{Simulation time resolution and horizon}

The model works for different time scale ranging from 1 minute to 1 hour time step with a time horizon hypothetically open, and is linked to the exogenous information input into the model - essentially the weather file. Input data regarding appliance usage is provided on an hourly basis and therefore down-scaling is necessary. This part is detailed in section 3 . 


\subsubsection{Electricity contracts}

Demand response programmes including real-time pricing, Time of Use tariffs, direct load control and other as listed in the literature (Pallonetto et al., 2020) are included in the model with the defined simulation time resolution. The dynamic pricing can further be limited upwards and downwards if necessary.

\subsection{Modelling framework}

The SBuM model is built into sub-modules that includes the power production, power consumption, heat demand, and control systems. The following sections briefly introduce the three main elements of the SBuM.

\subsubsection{Power consumption}

Power consumption is composed of 4 variables: electricity consumption for appliances, ventilation, heating/cooling elements, and domestic hot water. Power consumption distribution from appliances is described by Louis et al. (2016) and covers the fields of appliance modelling, user type considerations, demand response potential for appliances, and occupancy scenarios. Occupancy is deduced from the power consumption profiles from the appliances and not from occupancy profiles used as input to the model. This is to ensure that the power consumption from appliances and the active occupancy scenario are dependent and therefore coherent. Further on, the thermal needs of buildings were characterised to include a number of new variables that define the buildings physics and the ventilation technology used (Pulkkinen and Louis, 2019). Appliance specifics are given default values taken from the Remodece project (Almeida and Fonseca, 2006), but includes also other sources like the monitoring and research work from Murray et al. $(2016,2017)$ or Reinhardt et al. $(2012)^{2}$. The power module is and the occupancy is further used by the thermal demand model.

\subsubsection{Thermal demand}

The thermal demand of the model includes the thermal needs of the building and use thermal mass as a thermal storage to optimise the use of electricity when electric heating is used. The model is a white-box model, implemented by using the National Building Codes of Finland and the international standards on thermal behaviour such as ISO 52016-1, EN 15316-2, and the EN 15251. The interlink between the power module that covers all appliances and the thermal model is presented in Figure 3.

While the building physics are taken from the international standards, some added values are supplemented to address the issue of the internal gains, including human metabolism and appliance usage, as well as interconnecting the ventilation with the thermal losses and running of the heating system. The occupancy scenario, as defined in section 2.1.2, defines further the level of activities and allows recalculating the metabolism from the inhabitants. In

\footnotetext{
${ }^{2}$ http: / / www.tracebase.org
}

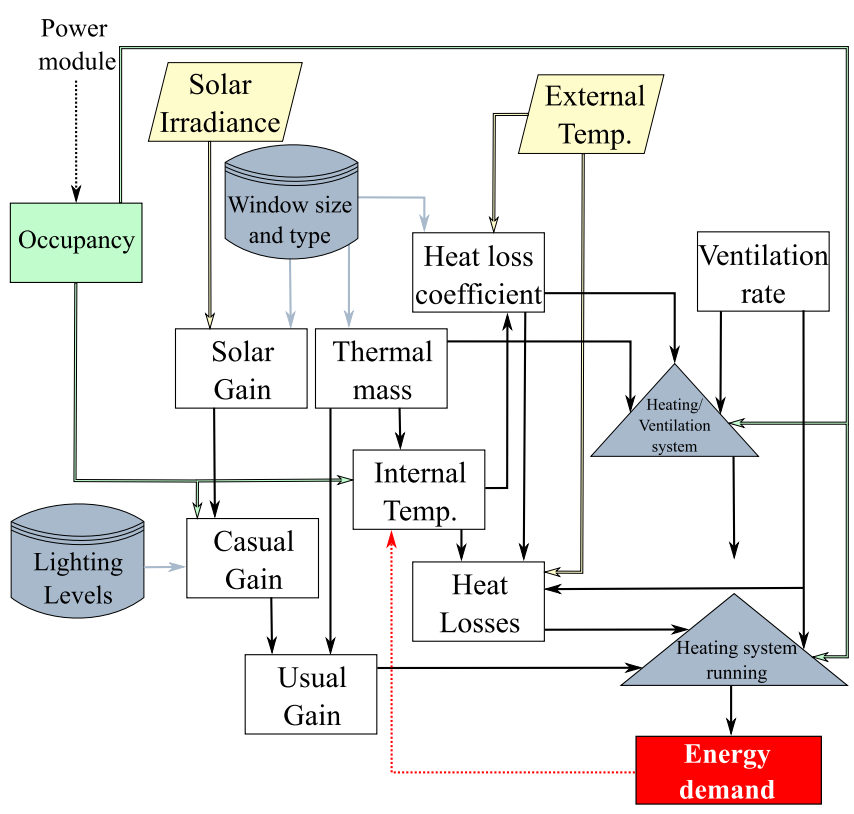

Figure 3. SBuM architecture connecting the power demand model and the heat demand model.

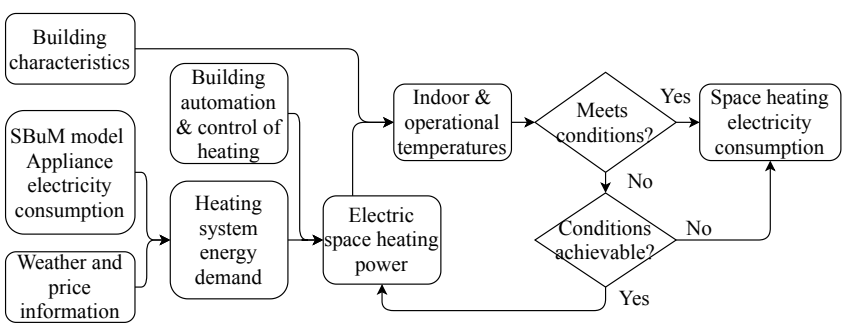

Figure 4. The operation of the electrical space heating part of the thermal model (Pulkkinen and Louis, 2019).

case of different type of space heating, the energy demand for each time resolution is provided and may potentially be used to recalculate the indoor temperature and electric heating power according to the predefined automation and control strategy. This operation principle is described in a flow sheet on the operation of the electric space heating part of the model presented in Figure 4 and in Pulkkinen and Louis (2019).

On-going work is being set up to include the use of domestic hot water provision from electric water tank .

\subsubsection{Optimisations}

The SBuM has a sub-block dedicated to the control system of the building and can consider multiple data input to optimise the consumption of the building. So far, two objectives are set, which are related to costs and environmental footprint of the consumed electricity. Secondly, the optimiser is only used when a home energy management system is setup for the studied house with the degree of control given to it. This means that the model can primarily be used to generate load profiles at a relatively high details, and can be consequently used in assessing optimisation control strategy based on dynamic pricing or other 
power network indicators. As the SBuM model is based on Markov-Chain, it is inherent to model consequential actions and therefore disrupt the power cycle from appliances or heating units.

Objective functions:

$$
\min p_{\text {total }, t}=\left[\left(\sum_{i=1}^{n} P_{i}\right)+P_{\text {heat }}+P_{\text {ventil }}\right] \times p_{t}
$$

where $p$ is the energy expenditure from the house $[€]$, and $P$ is the power consumption from all appliances $i$, heating and ventilation $[\mathrm{kWh}]$, and for each price at time $t$ $[€ / \mathrm{kWh}]$.

\subsection{Output data}

Four main variables are obtained from the model: the total power consumption from the building that can be disaggregated by appliances and heating modes, thermal performances of the building including indoor temperature variation, heat demand, thermal comfort and their associated variables, the environmental impact, granted that the electricity mix from the grid is known as a dynamic real-time environmental impact, and the cost of these implementations.

\section{Down-scaling simulation}

Handling multiple time resolution from input to output of the simulation is a challenge. The time resolution must be adapted for flexible input data, flexible time optimisation, and flexible time resolution of the output signal from the model.

For this reason, the model was made flexible to have any sort of inputs with a maximum time resolution of one hour and some pre-defined time resolution for the input database: 30-15-3-1 minutes and 10 seconds. These resolutions were taken arbitrarily to match the existing databases of appliance signatures (see section 2.2.1), weather data resolution, and power status from the transmission network (down to 3 minutes per sample).

\subsection{Scaling and multiplying}

Down-scaling power and heat demand profiles, can be performed either as disaggregated profile from the modelled profile or built up from lower timestep resolution and appliance signatures. Both ways provide output with a high granularity and can be used further in power system modelling. The advantage of proceeding power disaggregation is to perform faster simulation with a larger timestep e.g. 1 hour. The downside is that control cannot be performed at a lower resolution than the model itself and therefore demand response and control will be performed using the simulation steps and not the disaggregated granularity. The other way of implementing a high resolution model is to increase the simulation step of the simulation and thus provide better control for demand response actions based on price or power network status. The SBuM model allows carrying out both options with any simulation time. This is done by re-sampling the input dataset to meet the simulation time resolution and could be done simply by interpolating values, like in the case of temperature that does not vary very fast, or by performing linear interpolation and integrate a normally distributed noise to the dataset.

If a device is used for a very short time $\left(t_{c y c l, n}<t_{\min }\right)$, then the studied appliances $n$ could multiply its usage in order to comply with the average weekly usage of this device. This action multiplier only applies to new actions that has been triggered during the current iteration. Only in this condition the multiplying factor $t_{\text {mult }}$ can be generated, otherwise its value is set to 1 .

$$
\begin{aligned}
t_{\text {cycl }} & =t_{\text {mult }} \times t_{c y c l} \\
\text { where, } t_{\text {mult }} & =\left\lfloor R \sim U\left[1, \frac{t_{\text {min }}}{t_{\text {cycl }}}\right]\right\rfloor
\end{aligned}
$$

$T_{m u l t i}$ is a dimensionless value multiplying the number of actions within a time step when needed to reach the weekly average usage of appliances. The value of $T_{\text {multi }}$ is randomly generated and its value is comprised between a fraction of one cycle in comparison with the time step. The spread of the $T_{m u l t i}$ can be observed in Figure 5.

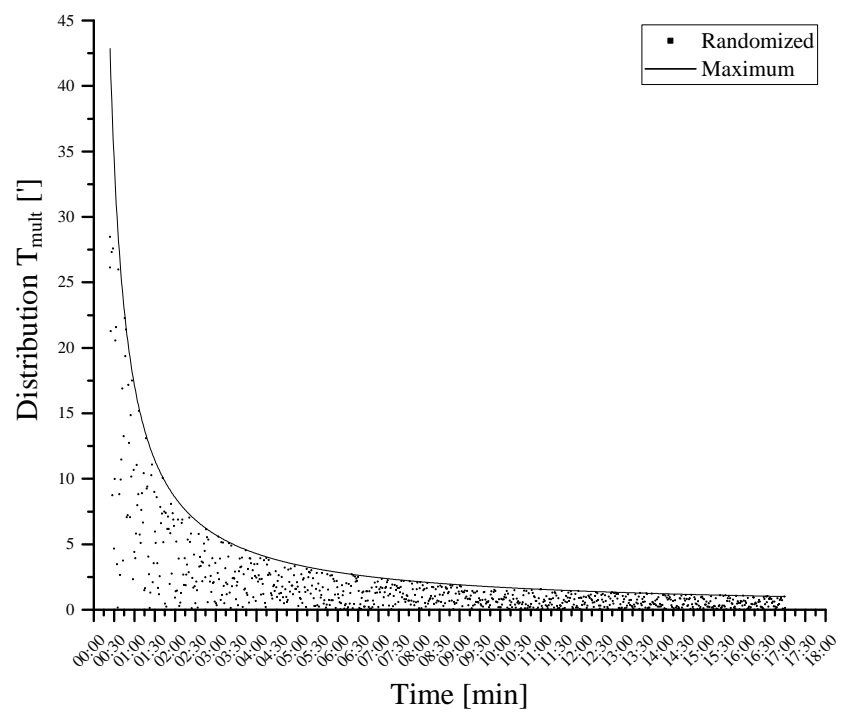

Figure 5. Distribution of $t_{\text {mult }}$ at multiple variable time usage $t_{c y c l}$ in hourly timestep simulation.

\section{Results and discussions}

The results of the SBuM model will be reviewed in terms of model performance in generating load and production profiles. These profiles are generated at different time scales through an event distribution throughout a typical day (Figure 6). This allows comparing the input distribution profile per appliance with the generated distribution profile for the same appliances. Generated load profiles are also looked through the prism of a detailed profile and comparing generated dataset at different granularity (Figure 7). Results are compared with models proposed to the 

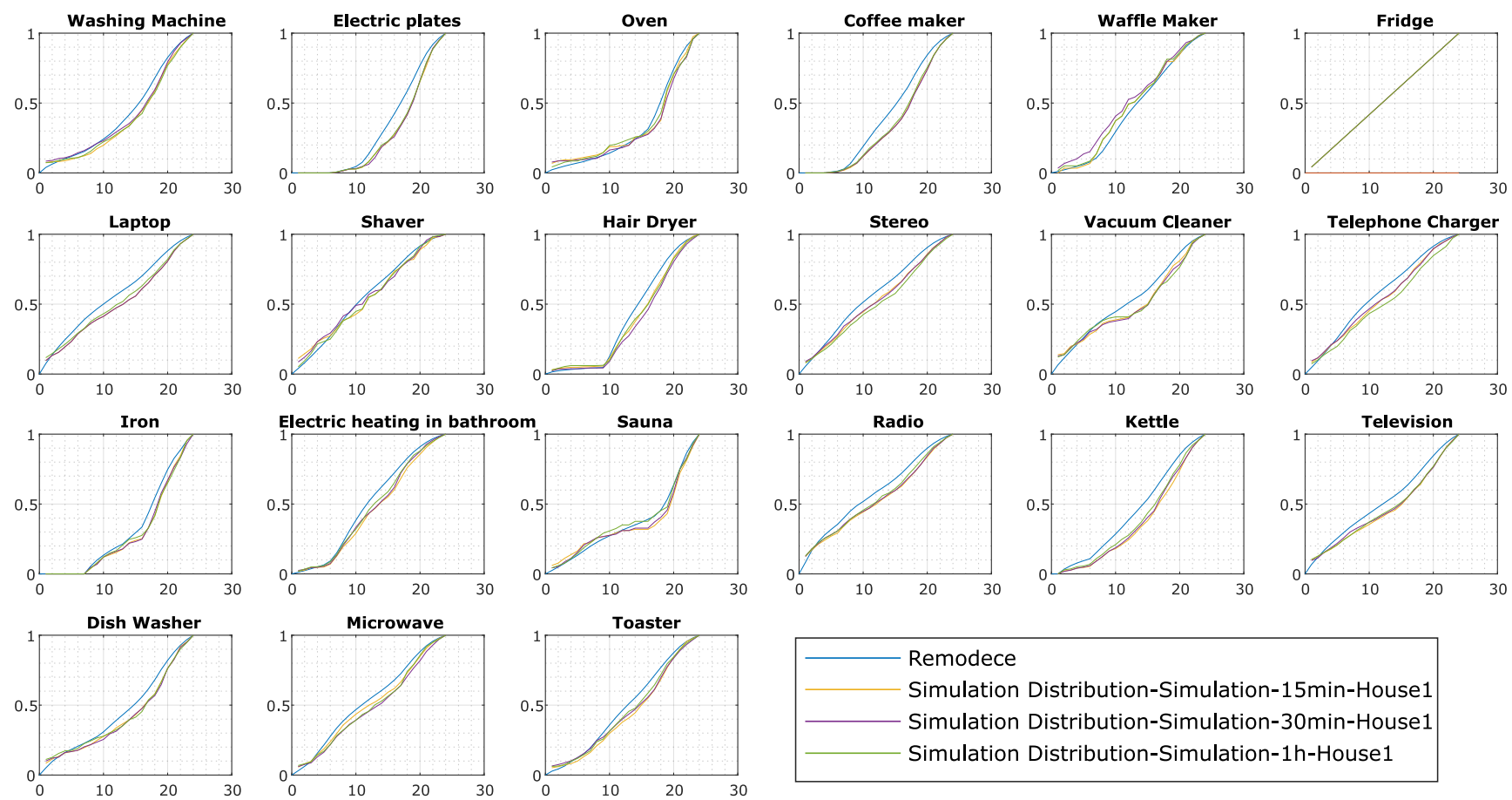

Figure 6. Cumulative daily distribution throughout the year against the REMODECE distribution used as input distribution in the SBuM model.

general public but do not allow high resolution modelling nor control options. For analysing the results, a set of 1year simulation was performed for a test reference year, from January $1^{\text {st }}$ to December $31^{\text {st }}, 2012$.

\subsection{Power demand}

The power demand of the buildings generated with the SBuM model will first be looked into the robustness to produce results similar to the input profiles given to the building, and second to the comparability levels of the different time resolution dataset produced while measuring their homogeneity through e.g. their daily load profiles.

\subsubsection{Power profile distribution}

Profile distributions for each appliance are taken from open dataset as described in section 2.2.1. The generated profiles are therefore easily comparable as their input profile are taken from the literature. A summary of 21 appliances used in the simulation is retrieved in Figure 6. The distribution profiles are aggregated to a daily load profile considering equally every day of the simulation (366 days).

Multiple statistical test were performed and for all appliances, the F-test are not statistically different at the 0.05 level, meaning that all distribution profile are statistically similar to the distribution dataset used for the appliances. This statistics is valid for all simulation granularity. In other words, the fit of distribution between the simulation output power profiles and the measured dataset of appliances measured in real living environment, demonstrate the robustness of the model and do not create unrealistic scenarios where appliances are run randomly.

\subsubsection{Dataset comparison}

Power demands from the appliances are depicted for different time scale simulations in Figure 7. The power profiles are put in perspective with the active occupancy scenario that are drawn from the power consumption profiles. As the simulations are independently run and rely on normally distributed random numbers under daily distribution constraints, the dataset with different granularity does not run the same scenario. Nevertheless, they do present similarities in their daily routines. The power disaggregatation of the 15 minutes time resolution into a 10 seconds profile is also shown on Figure 7.

Although the distribution of the appliances is done with an hourly distribution, smaller time resolution allows disrupting the hourly distribution profile and insert actions within these time-frames. The good correlation between the active occupation and the inactive occupation e.g. sitting, lying or else, is due to the fact that occupation is deduced from the appliance usage. This is critical for the good interrelation with the thermal model that calculates the thermal comfort and the exchange of heat within the building from human occupation.

The total power consumption for the different runs are consistent where the $1 \mathrm{~h}$ time resolution provides a power demand from appliances and lighting of $5050 \mathrm{kWh} / \mathrm{y}$, the 30 minutes granularity accounts for $5293 \mathrm{kWh} / \mathrm{y}$, and the 15 minutes resolution for $5158 \mathrm{kWh} / \mathrm{y}$. In this particular case, the consistency is of the annual power demand between time scales were a critical indicators. The dataset 


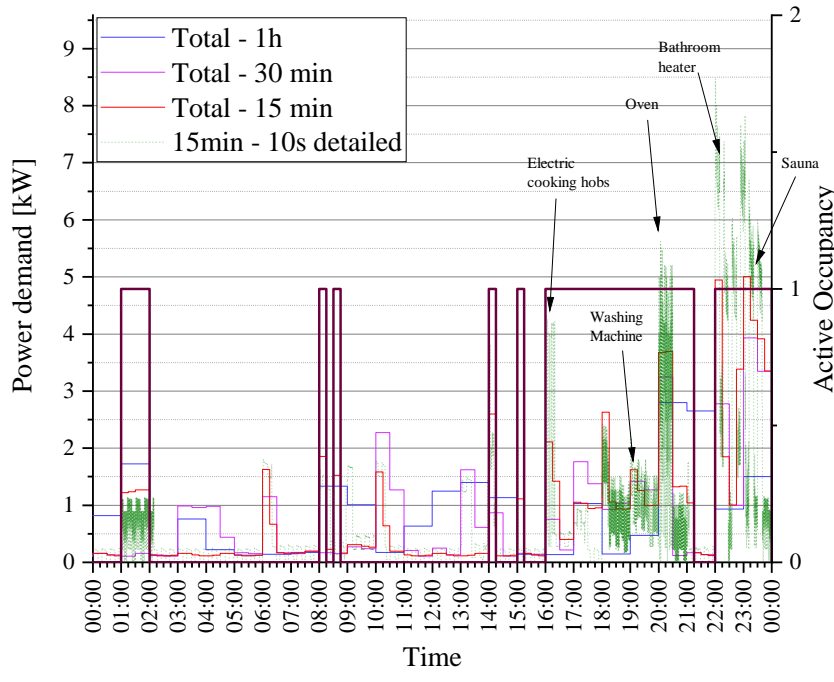

Figure 7. Energy service power load profiles and its occupancy for a typical summer day (1 day).

presents a similar mean value of power consumption for all time resolution simulations but with the higher granularity the power demand becomes more volatile which can be observed in the increase in their standard deviations and their associated coefficients of variation $(1.19,1.25,1.30$ for the 60 minutes, 30 minutes, and 15 minutes resolution respectively).

The daily load profile, which is intertwinked between the appliances power demand and their aggregation is depicted in Figure 8. The daily load profiles across the time

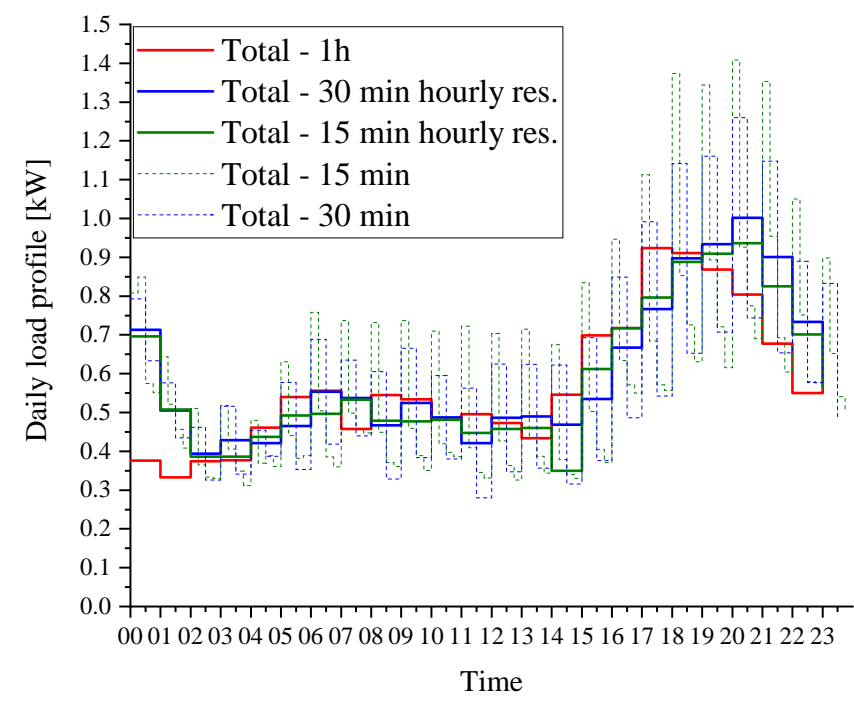

Figure 8. Mean daily load profiles generated by the 60/30/15 minutes time resolution simulations - one year simulation.

scales show good similarities that express their uniformity through the different runs of the model where the means for the $60 \mathrm{~min}, 30 \mathrm{~min}, 15 \mathrm{~min}$ are equal to $.57(\mathrm{SD}=.17$ ), $.60(\mathrm{SD}=.18)$, and $.59(\mathrm{SD}=.18) \mathrm{kW}$ respectively. Figure 8 also depicts the daily load profile of the same building that has a higher simulation time resolution, presenting therefore the higher variation. These variations are mainly due to the cycling of white appliances like the fridge that has a regular cycling time.

Downscaling the model time step to 10s allows fast control of some appliances if necessary. However, most of the benefits from existing demand side management (DSM) that reacts mainly on real time pricing that has been available at an hourly level so far. As there is intentions to decrease the resolution of day-ahead pricing to 15 minutes, it thus makes sense to be able to simulate buildings with this resolution and include their related controls. In any case, time resolution is always available at a 10 seconds resolution for the power module while the model may run with a larger time step, which can be meaningful for all sorts of engineering work. Furthermore, downscaling must be justified as it is a time consuming process where the computational time increases exponentially with the following relationship:

$$
y(x)=\frac{1}{7.04} \cdot e^{\left(\frac{0.9+x}{7.04}\right)}
$$

where $\mathrm{x}$ is the downscaling ratio. A full year simulation at an hourly time step takes about 6 minutes on a $32 \mathrm{~Gb}$ RAM and i7 core, while the same simulation with a time step of 1 minute may take over 80 hours with the same hardware.

\subsection{Thermal demand}

Thermal load is also detailed at the chosen time resolution and shows more discrepancies between the model resolution while staying within the constraints set in the simulation. The thermal performance of the building is analysed through the total heat demand in a year, the variation of the operative temperature (weighted average of the indoor and surface temperatures as defined in the ISO 52016-1), and the power demand in terms of power variation per degree change in indoor temperature before heating the building. The data are represented in Figure 9 for the coldest week performed in the simulation (from February $3^{\text {rd }}$ to February $\left.10^{\text {th }}\right)$. The temperature setting set in the simulation

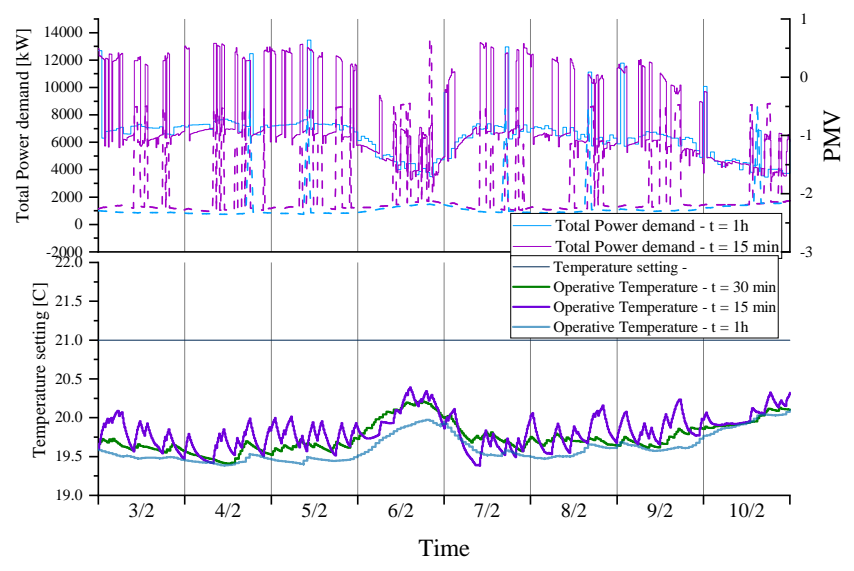

Figure 9. Indoor temperature variation and total thermal demand excluding DHW for the coldest week in the modelled year for three resolutions. Dotted lines represent the PMV variation for this same week 
is $21^{\circ} \mathrm{C}$ and as it can be seen in the Figure 9, the operative temperature is varying below this constraint. The mean operative temperature of the building is very similar in all 3 simulations, with a mean operative temperature of $20.4^{\circ} \mathrm{C}(\mathrm{SD}=.48), 20.5^{\circ} \mathrm{C}(\mathrm{SD}=.52)$, and $20.6^{\circ} \mathrm{C}(\mathrm{SD}=$ .7) for the $60 / 30 / 15$ minutes simulations respectively. The heat demand for the $60 / 30 / 15$ minutes simulation are 21.3 $\mathrm{MWh} / \mathrm{y}, 23.4 \mathrm{MWh} / \mathrm{y}$, and 24.4 MWh/y respectively. One of the reasons for difference of thermal energy consumption between the time resolutions is the shorter response time for adjusting the temperature within the building and keeping it to the correct level (adjusting the operative temperature to meet the temperature set for the house and therefore guaranteeing an indoor temperature of $21^{\circ} \mathrm{C}$ ).

The thermal comfort varies more in the negative values with an average of $-2(\mathrm{SD}=.28),-1.86(\mathrm{SD}=.45)$, and $-1.82(\mathrm{SD}=.5)$ for the $60 / 30 / 15$ minutes simulations respectively. Although the results indicate a cold feeling from the tenants $(<-1)$, the main reason resides in a paradoxical setting for the heating system that is controlled to keep the indoor temperature at $21^{\circ} \mathrm{C}$, and keeping the Predicted Mean Vote (PMV) level above (>) - .7 in the worst case. To reach the correct PMV level, a temperature setting of $23^{\circ} \mathrm{C}$ would be required but that would go against the Finnish regulation in its building code.

\subsection{PV generation}

The solar PV panel module presents results also for the different timestep sets for each simulation. The PVmodule is compared against results from PVGIS EUservice that provides hourly resolution for different types of PV systems. The PV model module was tested with the global irradiation array used in PVGIS with a system loss factor of $14 \%$, which includes dirt and performance decrease over time, which is fixed through the simulation. The nominal power of the PV-module is $10 \mathrm{~kW}$ in both settings. Both results are presented in Figure 10 top left graph for a typical summer week. While the datasets are statistically not different at $\mathrm{p}=.01$ during this extracted period and thus shows the good behaviour of the model during the summer months, the overall power generation from PV panels are different at $\mathrm{p}=.01$ for the overall year. In other words, the behaviour of the PV model is validated with some small discrepencies in the winter months resulting in different overall yearly energy production. The overall yearly power produced is however different with a total of $12.9 \mathrm{MWh} /$ year for the PVGIS model and 14.1 $\mathrm{MWh} /$ year for the SBuM model. The main difference resides in the winter month when the production is low, especially under high latitude where the simulations were performed $\left(65.05^{\circ}\right.$ North).

When running the simulation with more precise dataset from the Finnish Meteorological Institute (FMI), the power production has a greater Coefficient of Variation $(\mathrm{CV}=1.52$ for PVGIS and $\mathrm{CV}=1.84-1.9$ with FMI dataset) and this is due to the higher variation in the global irradiance dataset provided by FMI. The total production
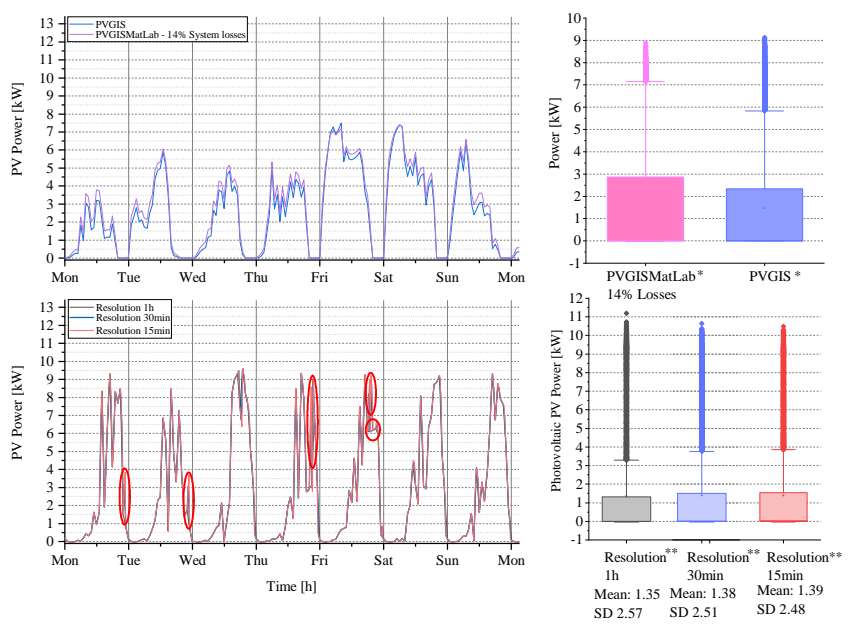

Figure 10. Top: Week production from a $10 \mathrm{~kW}$ PV system run with PVGIS online tool and the SBuM model using the PVGIS solar radiation data. Bottom: The SBuM model output using the Finnish Meteorological Institute solar radiation data for 3 time resolution, $1 \mathrm{~h}, 30$ minutes, and 15 minutes. *The population mean is significantly different at $\mathrm{p}=0.01$, **The population means are not significantly different at $\mathrm{p}=0.01$.

from the PV-panels using the FMI dataset is $11.9 \mathrm{MWh} / \mathrm{y}$, 12.1 MWh/y, and 12.2 MWh/y for the 60/30/15 minutes time resolution respectively. The model output is thus consistent with state of the art global output variables while providing more detailed information while the simulation resolution is increasing without altering the global output of the model, thus guaranteeing its use for automation purpose.

\section{Conclusions}

This paper presented a multi-scale open source modelling tool to evaluate the impact of smart control on the power and heat demand of the building. The SBuM model includes both the heat and power demand, as well as thermal comfort user satisfaction and willingness to shift load from appliances in case of energy management system. The model can be run at different time resolution from 60 minutes down to 10 seconds, therefore, providing a wide range of possible control strategies for distribution system operators as well as provide information to homeowners. As the SBuM model uses a Markov-Chain approach to the modelling of the buildings, the different energy points can be managed on the fly but post processing of the results can be done for further use. The real challenge was to create synthetic profiles that are comparable with different time scale resolutions and flexible in terms of computing, to be included in market operation as well as automation solutions.

The SBuM model showed robustness in the creation of load profiles for both thermal and power demands. The SBuM model generated profiles and datasets that were comparable and statistically not different across multiple time resolutions. However, the smaller the time resolution, the greater is the computing time of the simulations, 
therefore having a very low granularity in the simulation as it may take a very long time to compute when the simulation time horizon is set to a year for instance.

In the future, the SBuM model will integrate a model for domestic hot water integration with controllability in case it is electrically heated. It will therefore cover the whole range of energy consumption in dwellings. Further, the impact of different market configuration for dynamic pricing will be investigated to evaluate the impact of and on buildings. Heating control strategies will need to be implemented further to pilot electric convectors in a way that it does not increase the total energy consumption of the house.

\section{Acknowledgement}

This work was made possible through the University of Oulu strategic funding, the Energy as a Service Laboratory (EaaSLab) from the EU regional funding EAKR (A74849), the University of Oulu Graduate School (UniOGS). and the NEXUS4EU project of the Academy of Finland (decision number 333076) for participating to this conference.

\section{References}

Aníbal De Almeida and Paula Fonseca. Residential monitoring to decrease energy use and carbon emissions in Europe. International Energy ..., pages 1-14, 2006. URL http: //www.isr.uc.pt/ $\{\sim\}$ remodece/news/ Paper\{_\}DeAlmeida.pdf.

Drury B. Crawley, Linda K. Lawrie, Frederick C. Winkelmann, W.F. Buhl, Y.Joe Huang, Curtis O. Pedersen, Richard K. Strand, Richard J. Liesen, Daniel E. Fisher, Michael J. Witte, and Jason Glazer. EnergyPlus: creating a newgeneration building energy simulation program. Energy and Buildings, 33(4):319-331, Apr 2001. doi:10.1016/ s0378-7788(00)00114-6.

EU Commission. The european green deal, December 2019. URL https://eur-lex.europa. eu/legal-content/EN/ALL/?uri=CELEX: 52019DC0640.

Søren Østergaard Jensen, Anna Marszal-Pomianowska, Roberto Lollini, Wilmer Pasut, Armin Knotzer, Peter Engelmann, Anne Stafford, and Glenn Reynders. IEA EBC annex 67 energy flexible buildings. Energy and Buildings, 155:25-34, Nov 2017. doi:10.1016/j.enbuild.2017.08.044.

Jean-Nicolas Louis, Antonio Caló, Kauko Leiviskä, and Eva Pongrácz. Modelling home electricity management for sustainability: The impact of response levels, technological deployment \& occupancy. Energy and Buildings, 119:218-232, May 2016. doi:10.1016/j.enbuild.2016.03.012.

Antonio Luque and Steven Hegedus, editors. Handbook of Photovoltaic Science and Engineering. John Wiley \& Sons, Ltd, Dec 2010. doi:10.1002/9780470974704.

Eoghan McKenna and Murray Thomson. High-resolution stochastic integrated thermal-electrical domestic demand model. Applied Energy, 165:445-461, Mar 2016. doi:10.1016/j.apenergy.2015.12.089.

David Murray, Lina Stankovic, and Vladimir Stankovic. An electrical load measurements dataset of united kingdom households from a two-year longitudinal study. Scientific Data, 4(1), Jan 2017. doi:10.1038/sdata.2016.122.

D.M. Murray, J. Liao, L. Stankovic, and V. Stankovic. Understanding usage patterns of electric kettle and energy saving potential. Applied Energy, 171:231-242, Jun 2016. doi:10.1016/j.apenergy.2016.03.038.

Fabio Orecchini and Adriano Santiangeli. Beyond smart grids - the need of intelligent energy networks for a higher global efficiency through energy vectors integration. International Journal of Hydrogen Energy, 36(13):8126-8133, Jul 2011. doi:10.1016/j.ijhydene.2011.01.160.

Fabiano Pallonetto, Mattia De Rosa, Francesco D'Ettorre, and Donal P. Finn. On the assessment and control optimisation of demand response programs in residential buildings. Renewable and Sustainable Energy Reviews, 127:109861, Jul 2020. doi:10.1016/j.rser.2020.109861.

Jari Pulkkinen and Jean-Nicolas Louis. Utilizing flexibility of electric heating in demand side management programs in Finland in 2050. In Proceedings of 11th International Conference on Applied Energy, volume 4 (3), 2019.

Andreas Reinhardt, Paul Baumann, Daniel Burgstahler, Matthias Hollick, Hristo Chonov, Marc Werner, and Ralf Steinmetz. On the accuracy of appliance identification based on distributed load metering data. In Proceedings of the 2nd IFIP Conference on Sustainable Internet and ICT for Sustainability (SustainIT), 2012.

Melody Stokes. Removing barriers to embedded generation : a fine-grained load model to support low voltage network performance analysis. $\mathrm{PhD}$ thesis, Institute of Energy and Sustainable Development de Montfort University, Leicester, 2005. URL http: // hdl. handle. net/2086/4134.

Andong Wang, Rongling Li, and Shi You. Development of a data driven approach to explore the energy flexibility potential of building clusters. Applied Energy, 232:89-100, Dec 2018. doi:10.1016/j.apenergy.2018.09.187.

Selin Yilmaz, Steven K. Firth, and David Allinson. Occupant behaviour modelling in domestic buildings: the case of household electrical appliances. Journal of Building Performance Simulation, 10(5-6):582-600, Feb 2017. doi:10.1080/19401493.2017.1287775. 